\title{
Motivic intersection complex
}

\author{
by \\ Jörg Wildeshaus * \\ LAGA \\ UMR 7539 \\ Institut Galilée \\ Université Paris 13 \\ Avenue Jean-Baptiste Clément \\ F-93430 Villetaneuse \\ France \\ wildesh@math.univ-paris13.fr
}

April 28, 2011

\begin{abstract}
In this article, we give an unconditional definition of the motivic analogue of the intersection complex, establish its basic properties, and prove its existence in certain cases.
\end{abstract}

Keywords: Beilinson motives, motivic weight structure, Chow motives, motivic intersection complex.

Math. Subj. Class. (2010) numbers: 19E15 (14C25, 14F42, 14J17).

*Partially supported by the Agence Nationale de la Recherche, project no. ANR-07BLAN-0142 "Méthodes à la Voevodsky, motifs mixtes et Géométrie d'Arakelov". 


\section{Contents}

0 $\quad$ Introduction $\quad 2$

1 Review of weights on Beilinson motives 6

2 Definition of the motivic intersection complex 13

3 Basic properties $\quad 16$

4 On the problem of existence 21

\section{Introduction}

This paper contains largely extended notes of the talk the author gave during the conference Regulators III, which took place at the University of Barcelona in July 2010. Its main purpose is to propose an unconditional definition of the motivic intersection complex.

The use of the intersection complex, say in the context of (topological) sheaves on schemes over the complex numbers, or of ( $\ell$-adic) sheaves on schemes over a field, can be motivated by purity. Let $X$ be proper over $k$. Its singular cohomology (if $k=\mathbb{C}$ ) carries a pure Hodge structure, and its $\ell$-adic cohomology (if $k$ is finite or a number field) a pure Galois action, provided that $X$ is smooth. If this latter hypothesis is not met, then in order to get analogous purity statements, the constant sheaf on $X$ has to be replaced by the intersection complex [BBD] (with respect to the inclusion of the regular locus of $X$ ). Its (hyper)cohomology is known as intersection cohomology of $X$.

One of the main arithmetic applications to keep in mind concerns the Baily-Borel compactification of a smooth Shimura variety: it is canonical, and even minimal in a precise sense, but rarely smooth. Its intersection cohomology contains valuable arithmetic information, e.g., certain of its direct factors allow to realize Hodge structures and Galois representations associated to automorphic forms.

In order to construct motives inducing these Hodge structures and Galois representations via the respective realizations, one is thus led to try first to construct the intersection motive. One minimal requirement on this object would be that its realizations equal intersection cohomology. 
This construction succeeded in a small number of cases. Let us cite varieties (over $\mathbb{C}$ ) admitting semismall resolutions [CM], which includes the case of surfaces, and Baily-Borel compactifications of Hilbert-Blumenthal varieties [GHM] (over $\mathbb{C}$, and with more general than just constant coefficients). A general program for the construction of the intersection motive, assuming Grothendieck's standard conjectures, was developed (still over $\mathbb{C}$ ) in $[\mathrm{CH}]$.

When the construction works unconditionally, then it does so for specific geometric reasons. For example, such a reason would be that the relevant cycle classes are isomorphisms. The idea is basically to obtain an explicit formula for intersection cohomology sitting in the cohomology of a desingularization of $X$; the specific geometric reasons in question then allow to give a motivic sense to the explicit formula. Unfortunately, some functoriality properties valid for intersection cohomology are not a consequence of the explicit formula, and hence do not obviously hold for the intersection motive. This concerns for example the action of the Hecke algebra (which is needed in order to cut out the motive of an individual automorphic form from the intersection motive).

In [W1, we gave an unconditional construction of the intersection motive of Baily-Borel compactifications of smooth Hilbert-Blumenthal varieties with non-constant coefficients. It is a Chow motive over $\mathbb{Q}$, and behaves well under Hecke correspondences. Again, the construction works for specific geometric reasons, which translate into saying that "the boundary avoids weights -1 and 0 ". Let us not worry about the precise meaning of the "boundary" here. Rather, let us concentrate on the central notion of weight.

Assume first that our base scheme $X$ equals the spectrum of a perfect field $k$. According to Bondarko [Bo1], the category of geometrical motives VSF carries a weight structure, whose heart equals the category $C H M(k)$ of Chow motives over $k$. The precise definitions of weight structures and hearts will be recalled in the present Section 1; for the moment, let us keep in mind that the motivic weight structure allows for an intrinsic characterization of the full sub-category $C H M(k)$ of the category of geometrical motives. This is the key for everything to follow. Roughly speaking, the construction from [W1 works since geometrical motives are flexible enough to preserve functoriality; the problem of knowing whether the result of this construction is a Chow motive is then reduced to a computation of weights.

In general, the properties of intersection cohomology (functoriality, purity,...) are consequences of properties of the intersection complex. A general solution to the problem of constructing the intersection motive therefore requires the construction of the motivic intersection complex. Here, one is confronted with a foundational problem: the naïve generalization of the definition via truncations [BBD] cannot work since it requires the existence of 
a (perverse) $t$-structure. But even when the base is of the form Spec $k$, then except for certain fields $k$, such a $t$-structure is not known to exist on the category of geometrical motives. Thus, the mere problem of giving an unconditional definition of the motivic intersection complex is a priori non-trivial.

The solution to this problem that we shall propose, is again based on the notion of weight structure. In a way, our approach can be seen as "reading [BBD backwards", i.e., starting from [BBD, Chap. 5] on weights. This concerns in particular the Decomposition Theorem [BBD, Thm. 5.4.5], which implies that every pure complex on $X$ restricting to the structure sheaf on an open smooth sub-variety, contains the intersection complex as a direct factor. Let us indicate already here that the motivic analogue of this result (Theorem 3.1 (b)) is a rather elementary exercice in weight structures...

Let us now give a detailed overview of the individual sections of this paper. Section 1 starts with a review of Beilinson motives [CD], which conveniently generalize geometrical motives from Spec $k$ to arbitrary bases $X$. We then recall the basic notions related to weight structures. We review the main results from [Hé1 on the existence of the motivic weight structure on Beilinson motives (generalizing [Bo1] from Spec $k$ to $X$ ), and on the behaviour of weights under the six operations from CD. We then define the category $C H M(X)_{\mathbb{Q}}$ of Chow motives over $X$ as the heart of the motivic weight structure, and establish two complements of the theory. First (Theorem 1.7), we show that for an open sub-scheme $U$ of $X$, the inverse image from $C H M(X)_{\mathbb{Q}}$ to $C H M(U)_{\mathbb{Q}}$ is both essentially surjective and full. Following the terminology introduced in Bo2, this can be seen as a motivic version of resolution of singularities. Theorem 1.7 strenghens Bo2, Thm. 2.2.1 III 1], where essential surjectivity is proved up to pseudo-Abelian completion. Second (Theorem 1.12), we show that local duality respects the weights in a strict sense; in particular, the dual of a Chow motive is again a Chow motive. This complements [Hé2, Cor. 2.2.5], where the same result is proved provided $X$ is regular, and also [Hé1, Cor. 3.9], where left exactness (with respect to the weights) is established for any $X$.

Having in mind the Decomposition Theorem [BBD, Thm. 5.4.5], an intermediate extension of a Chow motive $M_{U}$ over a dense $U$ should satisfy a certain minimality condition among all possible extensions of $M_{U}$ to a Chow motive over $X$. In Section 2, we make this precise for regular $U$, and $M_{U}=\mathbb{1}_{U}$, the structure motive on $U$. More precisely (Definition 2.1), the motivic intersection complex $j_{! *} \mathbb{1}_{U}$ is a Chow motive on $X$ restricting to give $\mathbb{1}_{U}$, and admitting no non-trivial endomorphisms restricting trivially to $U$. We then establish independence of $j_{! *} \mathbb{1}_{U}$ of $U$ (Proposition 2.4). In its essence, it results from the study of a basic, but important example: when $X$ is regular, then $j_{! *} \mathbb{1}_{U}=\mathbb{1}_{X}$ (Example 2.3). 
Section 3 contains our main results. According to Theorem 3.1 (a), the motivic intersection complex is unique up to unique isomorphism. As already indicated, Theorem 3.1 (b) states that any extension of $\mathbb{1}_{U}$ to a Chow motive over $X$ contains $j_{!_{*}} \mathbb{1}_{U}$ as a direct factor - provided the latter exists. Under the same hypothesis, $j_{! *} \mathbb{1}_{U}$ is auto-dual (Corollary 3.8 ), meaning that the motivic intersection pairing can be defined. Theorem 3.11 identifies the few cases where we actually know the motivic intersection complex to exist. Section 4 contains the proof of Theorem 3.11

We choose to add a number of "Problems" in the text. While they concern properties that one might reasonably expect $j_{! *} \mathbb{1}_{U}$ to satisfy, the author does not know to solve any of them. The paper also contains a number of miscellaneous results, which are not needed elsewhere in the text, but seem worth to be mentioned nonetheless. In particular, this concerns Corollaries 1.10 and 3.5. The first (Corollary 1.10) states that for an open immersion $j: U \hookrightarrow X$ and any Chow motive $N_{U}$ over $U$, the image under the inverse image $j^{*}$ of motivic cohomology of $N$ in motivic cohomology of $N_{U}$ is independent of the extension of $N_{U}$ to a Chow motive $N$ over $X$. We relate this to Scholl's construction of "integral" sub-spaces of motivic cohomology of Chow motives over number fields (Remark 1.11). According to the second (Corollary [3.5), a Beilinson motive which is Nisnevich-locally isomorphic to $\mathbb{1}_{X}$, for a regular base $X$, is (globally) isomorphic to $\mathbb{1}_{X}$. This allows to generalize absolute purity [CD, Thm. 13.4.1] to arbitrary morphisms $a: X \rightarrow S^{\prime}$ between regular schemes: as soon as $a$ is of pure relative dimension $d$, there is an isomorphism $\mathbb{1}_{X}(d)[2 d] \cong a^{!} \mathbb{1}_{S^{\prime}}$ (Corollary [3.7).

Part of this work was done while I was enjoying a modulation de service pour les porteurs de projets de recherche, granted by the Université Paris 13, and during a stay at the University of Tokyo. I am grateful to both institutions. I wish to thank the organizers of Regulators III for the invitation to Barcelona, C. Soulé for a stimulating question asked during my talk, F. Déglise and D. Hébert for useful comments on a first draft of this paper, G. Ancona for strengthening an earlier version of Proposition 2.5, and the referee for her or his remarks and suggestions.

Conventions: Throughout the article, $S$ denotes a fixed base scheme, which we assume to be of finite type over an excellent scheme of dimension at most two. By definition, schemes are $S$-schemes which are separated and of finite type (in particular, they are excellent, and Noetherian of finite dimension), morphisms between schemes are separated morphisms of $S$-schemes, and a scheme is regular if the underlying reduced scheme is regular in the usual sense. 


\section{Review of weights on Beilinson motives}

We fix our base $S$, and work in the triangulated, $\mathbb{Q}$-linear categories $D M_{\mathrm{E}}(X)$ of Beilinson motives over $X$ [CD, Def. 13.2.1], indexed by schemes $X$ (always in the sense of the conventions fixed at the end of our Introduction). As in $\left[\mathrm{CD}\right.$, the symbol $\mathbb{1}_{X}$ is used to denote the unit for the tensor product in $D M_{\mathrm{5}}(X)$. We shall employ the full formalism of six operations developed in [loc. cit.]. Below, we shall list the principles (A)-(E) which will be particularly important to us. The global assumptions made in [loc. cit.] to establish these principles are met since $D M_{\mathrm{b}}(\bullet)$ is a motivic category [CD, Cor. 13.2.11], which by definition [CD, Def. 2.4.2] implies that it is pregeometric. Therefore, it is Sm-fibred [CD, Def. 1.1.9], the localization property $\left(L o c_{i}\right)$ from [CD, Def. 2.3.2] holds, and by [CD, Thm. 2.4.12] so do the proper transversality property from [CD, Def. 1.1.16] and the support property from [CD, Def. 2.2.5]. Furthermore, by [CD, Prop. 14.2.16], the category $D M_{\mathrm{E}}(\bullet)$ is separated in the sense of [CD, Def. 2.1.11]. By [CD, Ex. 14.3.20], it is pure in the sense of [CD, Def. 14.3.19]. (A) Absolute purity. Relation to K-theory: if $i: Z \hookrightarrow X$ is a closed immersion of pure codimension $c$ between regular schemes, then there is a canonical isomorphism

$$
\mathbb{1}_{Z}(-c)[-2 c] \stackrel{\sim}{\sim} i \mathbb{1}_{X}
$$

in $D M_{\mathrm{E}}(Z)[\mathrm{CD}$, Thm. 13.4.1]. For any regular scheme $X$, and any pair of integers $(p, q)$, there is a canonical isomorphism

$$
\operatorname{Hom}_{D M_{\mathrm{E}}(X)}\left(\mathbb{1}_{X}, \mathbb{1}_{X}(p)[q]\right) \cong \operatorname{Gr}_{\gamma}^{p} K_{2 p-q}(X)_{\mathbb{Q}},
$$

where $K .(X)_{\mathbb{Q}}$ denotes the tensor product of $K$-theory of $X$ with the rationals, and $\mathrm{Gr}_{\gamma}$ the graded object with respect to the (Adams) gamma filtration [CD, Cor. 13.2.14]. Furthermore, this isomorphism is contravariantly functorial with respect to morphisms of regular schemes [CD, Cor. 13.2.11]. (B) Base change: for any morphism $f$, there is a natural transformation

$$
\alpha_{f}: f_{!} \longrightarrow f_{*},
$$

which is an isomorphism is $f$ is proper [CD, Thm. 2.2.14 (1)]. If $f$ is the base of a cartesian diagram

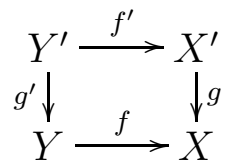

of schemes, then the exchange transformation

$$
g^{*} f_{!} \longrightarrow f_{!}^{\prime} g^{\prime *}
$$

is an isomorphism [CD, Prop. 2.2.13 (b)]. Hence so is the adjoint exchange transformation

$$
g_{*}^{\prime} f^{\prime !} \longrightarrow f^{!} g_{*}
$$


(C) Constructibility: by definition [CD, Def. 1.4.7], the full thick triangulated sub-category $D M_{\mathrm{E}, c}(X)$ of $D M_{\mathrm{E}}(X)$ of constructible objects is generated by the Tate twists $M_{X}(T)(p)$ of the motives $M_{X}(T)$ [CD, Sect. 1.1.33] of smooth $X$-schemes $T$. In particular, all twists $\mathbb{1}_{X}(p)$ belong to $D M_{\mathrm{L}, c}(X)$. By [CD, Ex. 14.1.3], an object of $D M_{\mathrm{L}}(X)$ is constructible if and only if it is compact. According to [CD, Thm. 14.1.31], the sub-categories $D M_{\mathrm{E}, c}(\bullet) \subset$ $D M_{\mathrm{E}}(\bullet)$ are respected by the six functors. (D) Duality: fix a scheme $X$ whose structure morphism to $S$ factors over a regular scheme; this is of course the case if $S$ is itself regular. According to [CD, Thm. 14.3.28], the category $D M_{\mathrm{E}, c}(X)$ then contains dualizing objects in the sense of [CD, Def. 14.3.10]. Fix such a dualizing object $R$. Define the local duality functor (with respect to $R$ ) as

$$
\mathbb{D}_{X}:=\underline{\operatorname{Hom}}_{X}(\bullet, R) .
$$

It is right adjoint to itself [CD, Sect. 14.3.30]. It preserves constructible objects, and the adjunction $\operatorname{id}_{X} \rightarrow \mathbb{D}_{X}^{2}$ is an isomorphism on $D M_{\mathrm{L}, c}(X)[\mathrm{CD}$, Cor. 14.3.31 (a), (b)]. Furthermore, it exchanges $f^{*}$ and $f^{!}$, as well as $f_{!}$and $f_{*}$ in the following sense: for a morphism $f: Y \rightarrow X$, put

$$
\mathbb{D}_{Y}:=\underline{\operatorname{Hom}}_{Y}\left(\bullet, f^{!} R\right) ;
$$

note that according to [CD, Prop. 14.3.29 (ii)], the motive $f^{!} R$ is dualizing on $Y$. Then there are natural isomorpisms of functors

$$
\mathbb{D}_{Y} f^{*} \stackrel{\sim}{\longrightarrow} f^{!} \mathbb{D}_{X} \text { and } f_{*} \mathbb{D}_{Y} \stackrel{\sim}{\longrightarrow} \mathbb{D}_{X} f_{!}
$$

on $D M_{\mathrm{E}}(\bullet)$ CD, Cor. 14.3.31 (d) and its proof]. Therefore,

$$
f^{*} \mathbb{D}_{X} \stackrel{\sim}{\longrightarrow} f^{!} \mathbb{D}_{Y} \text { and } \mathbb{D}_{X} f_{*} \stackrel{\sim}{\longrightarrow} f_{!} \mathbb{D}_{Y}
$$

on $D M_{\mathrm{E}, c}(\bullet)$. For the applications of duality that we have in mind, we need to make explicit choices of dualizing object $R$. Fix a pair of integers $(p, q)$, and a morphism $a: X \rightarrow S^{\prime}$ with regular target. Then

$$
R:=a^{!} \mathbb{1}_{S^{\prime}}(p)[q] \in D M_{\mathrm{E}, c}(X)
$$

is a dualizing object $[\mathrm{CD}$, Prop. 14.3.29]. It will be necessary to identify $R$ under the following additional hypotheses on $X$ : the morphism $a: X \rightarrow S^{\prime}$ is quasi-projective, and $X$ is regular and connected of relative dimension $e$ over $S^{\prime}$. We claim that in this case, there is an isomorphism

$$
R \stackrel{\sim}{\longrightarrow} \mathbb{1}_{X}(p+e)[q+2 e] .
$$

Indeed, absolute purity (see point (A)) and the formula $j^{!}=j^{*}$ for an open immersion $j$ CD, Thm. 2.2.14 (2)] reduces us to the case when $X$ is a projective space over $S^{\prime}$. Our claim then follows from Ay, Scholie 1.4.2 3] (via [CD, Cor. 2.4.9]). (E) Localization: if $i: Z \hookrightarrow X$ and $j: U \hookrightarrow X$ are complementary closed, resp. open immersions of schemes, then there are canonical exact triangles

$$
j_{!} j^{*} \longrightarrow \operatorname{id}_{X} \longrightarrow i_{*} i^{*} \longrightarrow j ! j^{*}[1]
$$




$$
i_{*} i^{!} \longrightarrow \mathrm{id}_{X} \longrightarrow j_{*} j^{*} \longrightarrow i_{*} i^{!}[1]
$$

of exact endo-functors of $D M_{\mathrm{E}}(X)$ [CD, Prop. 2.3 .3 (2), (3), Thm. 2.2.14 (2)]. The adjunctions $\operatorname{id}_{U} \rightarrow j^{*} j_{!}, j^{*} j_{*} \rightarrow \mathrm{id}_{U}$ and $i^{*} i_{*} \rightarrow \mathrm{id}_{Z}$ are isomorphisms, and the compositions $i^{*} j_{\text {! }}$ and $j^{*} i_{*}$ are trivial [CD, Sect. 2.3.1]. From what precedes, it follows formally that the adjunction $\operatorname{id}_{Z} \rightarrow i^{i} i_{*}$ is an isomorphism, and that the composition $i^{!} j_{*}$ is trivial. We also see, putting $i$ equal to the immersion of the reduced scheme structure $X_{\text {red }}$ on $X$, that

$$
i_{*}: D M_{\mathrm{E}}\left(X_{\text {red }}\right) \longrightarrow D M_{\mathrm{E}}(X)
$$

is an equivalence of categories, with canonical quasi-inverse $i^{!}=i^{*}$. This jusitifies a posteriori the abuse of language fixed in the conventions at the end of our Introduction.

Now recall the following notions, due to Bondarko.

Definition 1.1 ([Bo1, Def. 1.1.1]). Let $\mathcal{C}$ be a triangulated category. A weight structure on $\mathcal{C}$ is a pair $w=\left(\mathcal{C}_{w \leq 0}, \mathcal{C}_{w \geq 0}\right)$ of full sub-categories of $\mathcal{C}$, such that, putting

$$
\mathcal{C}_{w \leq n}:=\mathcal{C}_{w \leq 0}[n] \quad, \quad \mathcal{C}_{w \geq n}:=\mathcal{C}_{w \geq 0}[n] \quad \forall n \in \mathbb{Z},
$$

the following conditions are satisfied.

(1) The categories $\mathcal{C}_{w \leq 0}$ and $\mathcal{C}_{w \geq 0}$ are Karoubi-closed: for any object $M$ of $\mathcal{C}_{w \leq 0}$ or $\mathcal{C}_{w \geq 0}$, any direct summand of $M$ formed in $\mathcal{C}$ is an object of $\mathcal{C}_{w \leq 0}$ or $\mathcal{C}_{w \geq 0}$, respectively.

(2) (Semi-invariance with respect to shifts.) We have the inclusions

$$
\mathcal{C}_{w \leq 0} \subset \mathcal{C}_{w \leq 1} \quad, \quad \mathcal{C}_{w \geq 0} \supset \mathcal{C}_{w \geq 1}
$$

of full sub-categories of $\mathcal{C}$.

(3) (Orthogonality.) For any pair of objects $A \in \mathcal{C}_{w \leq 0}$ and $B \in \mathcal{C}_{w \geq 1}$, we have

$$
\operatorname{Hom}_{\mathcal{C}}(A, B)=0 \text {. }
$$

(4) (Weight filtration.) For any object $M \in \mathcal{C}$, there exists an exact triangle

$$
A \longrightarrow M \longrightarrow B \longrightarrow A[1]
$$

in $\mathcal{C}$, such that $A \in \mathcal{C}_{w \leq 0}$ and $B \in \mathcal{C}_{w \geq 1}$.

Slightly generalizing the above terminology, for $n \in \mathbb{Z}$, we shall refer to any exact triangle

$$
A \longrightarrow M \longrightarrow B \longrightarrow A[1]
$$

in $\mathcal{C}$, with $A \in \mathcal{C}_{w \leq n}$ and $B \in \mathcal{C}_{w \geq n+1}$, as a weight filtration of $M$. 
Definition 1.2 ([Bo1, Def. 1.2.1 1]). Let $w$ be a weight structure on $\mathcal{C}$. The heart of $w$ is the full additive sub-category $\mathcal{C}_{w=0}$ of $\mathcal{C}$ whose objects lie both in $\mathcal{C}_{w \leq 0}$ and in $\mathcal{C}_{w \geq 0}$.

Beilinson motives can be endowed with weight structures, thanks to the main results from [Hé1]. More precisely, the following holds.

Theorem 1.3 ([Hé1, Thm. 3.3, Thm. 3.8 (i)-(ii)]). (a) There are canonical weight structures $w$ on the categories $D M_{\mathrm{E}, c}(\bullet)$. They are uniquely characterized by the following properties.

(a1) The objects $\mathbb{1}_{X}(p)[2 p]$ belong to the heart $D M_{\mathrm{E}, c}(X)_{w=0}$, for all integers $p$, whenever $X$ is regular.

(a2) For a morphism of schemes $f$, left adjoint functors $f^{*}, f_{\text {! }}$ and $f_{\sharp}$ (the latter for smooth $f$ ) are $w$-left exact, i.e., they map $D M_{\mathrm{E}, c}(\bullet)_{w \leq 0}$ to $D M_{\mathrm{L}, c}(\bullet)_{w \leq 0}$, and right adjoint functors $f_{*}, f^{!}$and $f^{*}$ (the latter for smooth $f$ ) are $w$-right exact, i.e., they $\operatorname{map} D M_{\mathrm{E}, c}(\bullet)_{w \geq 0}$ to $D M_{\mathrm{E}, c}(\bullet)_{w \geq 0}$.

(b) There are canonical weight structures $W$ on the categories $D M_{\mathrm{B}}(\bullet)$. They induce the weight structures $w$ on the categories $D M_{\mathrm{E}, c}(\bullet)$. They are uniquely characterized by the requirement that any small sum of objects of $D M_{\mathrm{E}, c}(X)_{w=0}$ lie in $D M_{\mathrm{E}}(X)_{W=0}$.

Let us refer to the weight structure $w$ on $D M_{\mathrm{B}, c}(\bullet)$ as the motivic weight structure. Theorem 1.3 generalizes an earlier result of Bondarko's Bo1, Prop. 6.5.3] concerning the case $X=$ Spec $k$, for a perfect field $k$ (use [CD, Rem. 10.1.5, Thm. 15.1.4] to get the equivalence between the triangulated category of geometrical motives à la Voevodsky and $\left.D M_{\mathrm{E}, c}(\mathbf{S p e c} k)\right)$.

Remark 1.4. Since the first appearance of [Hé1, a different proof of existence of the motivic weight structure was given in [Bo2, Thm. 2.1.1]. The $w$-exactness properties from [Hé1, Thm. 3.8] are shown in [Bo2, Thm. 2.2.1 II] for quasi-projective morphisms of schemes. The results of [Bo2] were obtained independently from [Hé1].

Note that for perfect fields $k$, [Bo1, Sect. 6.6] allows to identify the heart of the motivic weight structure on $D M_{\mathrm{Б}, c}(\operatorname{Spec} k)$ with the category (opposite to the category) of Chow motives over $k$. This motivates the following.

Definition 1.5. The $\mathbb{Q}$-linear category $C H M(X)_{\mathbb{Q}}$ of Chow motives over $X$ is defined as the heart $D M_{\mathrm{E}, c}(X)_{w=0}$ of the motivic weight structure.

Remark 1.6. The categories $D M_{\mathrm{L}, c}(\bullet)$ are pseudo-Abelian (see [Hé1, Sect. 2.10]). Hence so are their hearts $C H M(\bullet)_{\mathbb{Q}}$. For a fixed scheme $X$, the category $C H M(X)_{\mathbb{Q}}$ can be constructed as the pseudo-Abelian completion of the category of motives over $X$ of the form

$$
f_{!} \mathbb{1}_{Y}(p)[2 p],
$$


for proper morphisms $f: Y \rightarrow X$ with regular source $Y$, and integers $p$ [é1, Thm. 3.3 (ii)]. Since by [CD, Cor. 14.3.9] these motives generate $D M_{\mathrm{L}, c}(X)$ as a thick triangulated category, we see in particular that the latter is generated by the heart of its weight structure.

Here is our first application of the formalism of motivic weight structures.

Theorem 1.7. Let $j: U \hookrightarrow X$ be an open immersion of schemes.

(a) The inverse image

$$
j^{*}: C H M(X)_{\mathbb{Q}} \longrightarrow C H M(U)_{\mathbb{Q}}
$$

is essentially surjective.

(b) The inverse image $j^{*}$ is full.

Note that by Theorem 1.3 (a2), the functor $j^{*}$ is $w$-exact, meaning that it is both $w$-left and $w$-right exact ( $j$ is smooth). In particular, it preserves the hearts of the weight structures on $D M_{\mathrm{E}, c}(X)$ and on $D M_{\mathrm{L}, c}(U)$. Note also that essential surjectivity of $j^{*}$ on both $D M_{\mathrm{Б}, c}(\bullet)_{w \leq 0}$ and $D M_{\mathrm{E}, c}(\bullet)_{w \geq 0}$ is a formal consequence of the existence of $j_{\text {! }}$ and $j_{*}$, and the formulae $\mathrm{id}_{U} \cong j^{*} j_{\text {! }}$ and $j^{*} j_{*} \cong \mathrm{id}_{U}$. (By contrast, $j^{*}$ should not in general be expected to be full on $D M_{\mathrm{E}, c}(\bullet)_{w \leq 0}$ or on $D M_{\mathrm{b}, c}(\bullet)_{w \geq 0}$ !) Theorem 1.7 (a) strenghens [Bo2, Thm. 2.2.1 III 1 ], where it is proved that $j^{*}$ is essentially surjective up to pseudo-Abelian completion.

Proof of Theorem 1.7. (a) Let $M_{U}$ be an object of $C H M(U)_{\mathbb{Q}}$, and consider the morphism

$$
m:=\alpha_{j}\left(M_{U}\right): j_{!} M_{U} \longrightarrow j_{*} M_{U}
$$

of motives over $X$ (see point (B) above). Applying $j^{*}$ to $m$ yields an isomorphism. Therefore, by localization, any cone of $m$ is of the form $i_{*} C$, for a motive $C$ over the complement $i: Z \hookrightarrow X$ of $U$ in $X$ (with the reduced scheme structure, say). Choose and fix such a cone $i_{*} C$, as well as a weight filtration

$$
C_{\leq 0} \stackrel{c_{-}}{\longrightarrow} C \stackrel{c_{+}}{\longrightarrow} C_{\geq 1} \stackrel{\delta}{\longrightarrow} C_{\leq 0}[1]
$$

of $C \in D M_{\mathrm{E}, c}(Z)$ (Theorem $1.3(\mathrm{a})$ ). Thus,

$$
C_{\leq 0} \in D M_{\mathrm{E}, c}(Z)_{w \leq 0} \quad \text { and } \quad C_{\geq 1} \in D M_{\mathrm{E}, c}(Z)_{w \geq 1} .
$$

According to axiom TR4' of triangulated categories (see [BBD, Sect. 1.1.6] 
for an equivalent formulation), the diagram of exact triangles

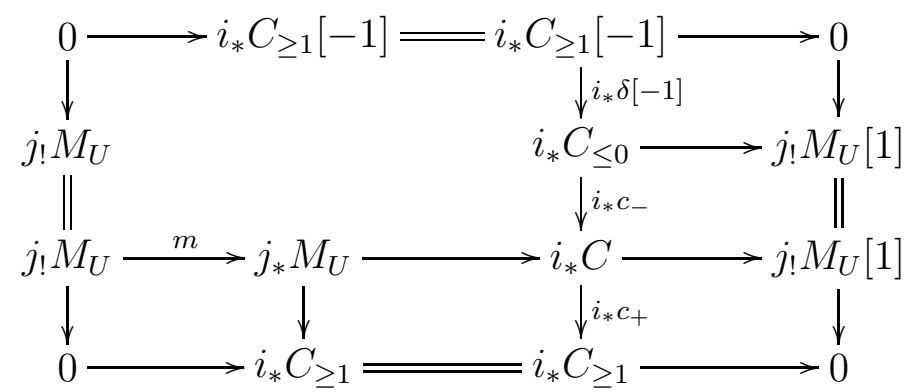

in $D M_{\mathrm{Б}, c}(X)$ can be completed to give

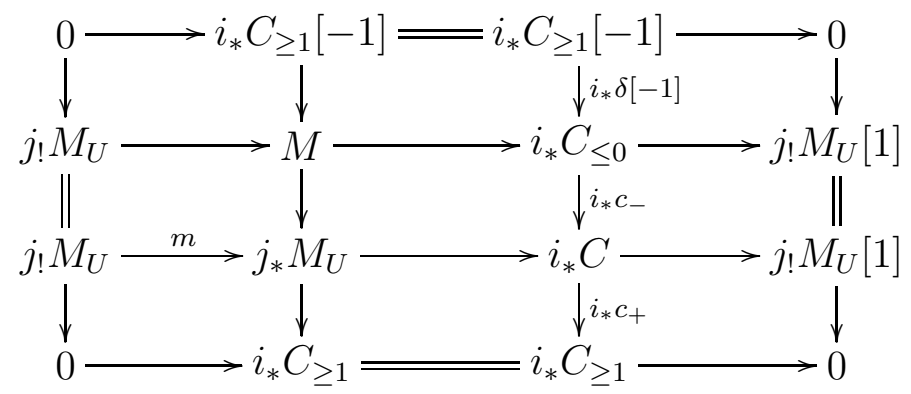

with $M \in D M_{\mathrm{E}, c}(X)$. Since the composition of functors $j^{*} i_{*}$ is trivial, the inverse image $j^{*} M$ is isomorphic to $M_{U}$. Now observe that by Theorem1.3 (a2), the functors $i_{!}=i_{*}$ and $j_{!}$are $w$-left exact, and $i_{*}$ and $j_{*}$ are $w$-right exact. Thus, by the above diagram, the motive $M$ is simultaneously an extension of motives of weights $\leq 0$, and an extension of motives of weights $\geq 0$. It follows easily (see [Bo1, Prop. 1.3.3 3]) that $M$ is pure of weight zero.

(b) Now let $M$ and $N$ be Chow motives over $X$, and assume that a morphism

$$
\beta_{U}: j^{*} M \longrightarrow j^{*} N
$$

between their restrictions to $U$ is given. Consider the localization triangles for $M$ and for $N$.

$$
\begin{aligned}
& i_{*} i^{*} M[-1] \longrightarrow j_{!} j^{*} M \longrightarrow \\
& \downarrow_{j !} \beta_{U} \\
& i_{*} i^{*} N[-1] \longrightarrow i_{*} i^{*} M \\
& j_{!} j^{*} N \longrightarrow
\end{aligned}
$$

According to Theorem 1.3 (a2), they provide weight filtrations of $j_{!} j^{*} M$ and of $j_{!} j^{*} N$, respectively. By orthogonality (condition (3) in Definition 1.1), any morphism from $i_{*} i^{*} M[-1]$ to $N$ is zero. Therefore, the above diagram can be completed to give a morphism of exact triangles.

q.e.d.

Remark 1.8. Following the lines of part (a) of the above proof, one can show that there is in fact a canonical bijection between the isomorphism classes of extensions of $M_{U}$ to $X$ as Chow motives on the one hand, and 
isomorphism classes of weight filtrations of the restriction of a cone of $j_{!} M_{U} \rightarrow$ $j_{*} M_{U}$ to the complement $X-U$ on the other hand.

Let us note a consequence of Theorem 1.7, which we think of as useful even though it will not be used in the rest of this paper.

Corollary 1.9. Let $j: U \hookrightarrow X$ be an open immersion of schemes. Let $N_{U}^{1}, N_{U}^{2} \in C H M(U)_{\mathbb{Q}}$ and $M^{1}, M^{2} \in D M_{\mathrm{E}}(X)$. Then the image of the inverse image

$j^{*}: \operatorname{Hom}_{X}\left(M^{1} \otimes_{X} N^{1}, M^{2} \otimes_{X} N^{2}\right) \longrightarrow \operatorname{Hom}_{U}\left(j^{*} M^{1} \otimes_{U} N_{U}^{1}, j^{*} M^{2} \otimes_{U} N_{U}^{2}\right)$ is independent of the extensions of $N_{U}^{n}$ to Chow motives $N^{n}$ over $X, n=1,2$.

Proof. Let $N_{r}^{n} \in C H M(X)_{\mathbb{Q}}, r=1,2$ be two extensions of $N_{U}^{n}, n=$ 1,2. By Theorem 1.7 (b), there are morphisms $\beta_{1}^{n}: N_{1}^{n} \rightarrow N_{2}^{n}$ and $\beta_{2}^{n}$ : $N_{2}^{n} \rightarrow N_{1}^{n}$ extending id $N_{U}^{n}$. But then,

$j^{*}: \operatorname{Hom}_{X}\left(M^{1} \otimes_{X} N_{1}^{1}, M^{2} \otimes_{X} N_{1}^{2}\right) \longrightarrow \operatorname{Hom}_{U}\left(j^{*} M^{1} \otimes_{U} N_{U}^{1}, j^{*} M^{2} \otimes_{U} N_{U}^{2}\right)$

factors through $\operatorname{Hom}_{X}\left(M^{1} \otimes_{X} N_{2}^{1}, M^{2} \otimes_{X} N_{2}^{2}\right)$, and

$j^{*}: \operatorname{Hom}_{X}\left(M^{1} \otimes_{X} N_{2}^{1}, M^{2} \otimes_{X} N_{2}^{2}\right) \longrightarrow \operatorname{Hom}_{U}\left(j^{*} M^{1} \otimes_{U} N_{U}^{1}, j^{*} M^{2} \otimes_{U} N_{U}^{2}\right)$

factors through $\operatorname{Hom}_{X}\left(M^{1} \otimes_{X} N_{1}^{1}, M^{2} \otimes_{X} N_{1}^{2}\right)$.

q.e.d.

Corollary 1.10. Let $j: U \hookrightarrow X$ be an open immersion of schemes. Let $N_{U} \in C H M(U)_{\mathbb{Q}}$ and $(p, q) \in \mathbb{Z}^{2}$. Then the image of the inverse image

$$
j^{*}: \operatorname{Hom}_{X}\left(\mathbb{1}_{X}, N(p)[q]\right) \longrightarrow \operatorname{Hom}_{U}\left(\mathbb{1}_{U}, N_{U}(p)[q]\right)
$$

is independent of the extension of $N_{U}$ to a Chow motive $N$ over $X$.

Remark 1.11. Corollary 1.10 should be compared to Scholl's construction of "integral" sub-spaces of motivic cohomology for Chow motives over local and global fields [S, Sect. 1]. In fact, continuity [CD, Thm. 14.2.5] implies that both statements of Theorem 1.7 continue to hold when passing to the limit over all open sub-schemes of a given scheme $X$. In particular, for any Dedekind domain $A$ with fraction field $K$, the restriction from $C H M(\operatorname{Spec} A)_{\mathbb{Q}}$ to $C H M(\operatorname{Spec} K)_{\mathbb{Q}}$ is essentially surjective and full. This yields the categorial interpretation of [S, Sect. 1]. It also shows that Scholl's construction generalizes to the inclusion of a generic point of any scheme $X$ (always in the sense of our conventions), which may thus be chosen differently from the spectrum of a Dedekind domain.

We finish this section with a discussion of the behaviour of weights under duality. Fix $X$, and suppose that the structure morphism of $X$ factors over a morphism $a: X \rightarrow S^{\prime}$ with regular target. Fix an integer $d$, put

$$
R:=a^{!} \mathbb{1}_{S^{\prime}}(-d)[-2 d] \in D M_{\mathrm{Б}, c}(X),
$$


and form the local duality functor $\mathbb{D}_{X}$ with respect to this choice of $R$ (see point (D) above). Part (a) of the following is contained in [Hé1, Cor. 3.9]; statements (a)-(c) are proved for regular $X$ in [Hé2, Cor. 2.2.5].

Theorem 1.12. Let $n$ be an integer, and consider the functor

$$
\mathbb{D}_{X}: D M_{\mathrm{E}, c}(X)^{\text {opp }} \longrightarrow D M_{\mathrm{E}, c}(X) \text {. }
$$

(a) $\mathbb{D}_{X}$ maps $D M_{\mathrm{E}, c}(X)_{w \leq n}^{o p p}$ to $D M_{\mathrm{E}, c}(X)_{w \geq-n}$.

(b) $\mathbb{D}_{X}$ maps $D M_{\mathrm{E}, c}(X)_{w \geq n}^{o p p}$ to $D M_{\mathrm{E}, c}(X)_{w \leq-n}$.

(c) $\mathbb{D}_{X}$ maps $C H M(X)_{\mathbb{Q}}{ }^{\text {opp }}$ to $C H M(X)_{\mathbb{Q}}$.

Given that $\mathrm{id}_{X}=\mathbb{D}_{X}^{2}$ on $D M_{\mathrm{L}, c}(X)$, we see that $\mathbb{D}_{X}$ actually induces equivalences of categories $D M_{\mathrm{B}, c}(X)_{w \leq n}^{o p p} \cong D M_{\mathrm{E}, c}(X)_{w \geq-n}$ etc.

Proof of Theorem 1.12. The thick triangulated category $D M_{\mathrm{E}, c}(X)$ is generated by its heart $C H M(X)_{\mathbb{Q}}$, and $\mathbb{D}_{X}$ inverts the sign of the shifts. Therefore, it suffices to prove part (c). By Hé1, Thm. 3.3 (ii)] (see Remark (1.6), it is enough to prove that for any proper morphism $f: Y \rightarrow X$ with regular source $Y$, and any integer $p$, the constructible Beilinson motive

$$
\mathbb{D}_{X}\left(f_{!} \mathbb{1}_{Y}(p)[2 p]\right)
$$

is actually a Chow motive. From the formulae recalled in point (D) above,

$$
\mathbb{D}_{X}\left(f_{!} \mathbb{1}_{Y}(p)[2 p]\right) \cong f_{!} \mathbb{D}_{Y}\left(\mathbb{1}_{Y}(p)[2 p]\right)
$$

(recall that $f$ is proper), provided $\mathbb{D}_{Y}$ is formed with respect to $f^{!} R$. But $f^{!} R=(a \circ f)^{!} \mathbb{1}_{S^{\prime}}(-d)[-2 d]$, hence

$$
\mathbb{D}_{Y}\left(\mathbb{1}_{Y}(p)[2 p]\right) \cong(a \circ f)^{!} \mathbb{1}_{S^{\prime}}(-(d+p))[-2(d+p)] .
$$

$Y$ has a finite Zariski covering by connected quasi-projective schemes $Y_{i}$ over $S^{\prime}$. Therefore (still thanks to point (D) above), the restriction to any $Y_{i}$ of the motive $(a \circ f)^{!} \mathbb{1}_{S^{\prime}}(-(d+p))[-2(d+p)]$ is isomorphic to $\mathbb{1}_{Y_{i}}(m)[2 m]$, for some integer $m$. In particular, we see that $\mathbb{D}_{Y}\left(\mathbb{1}_{Y}(p)[2 p]\right)$ is Zariski-locally of weight zero. The two localization triangles, together with the $w$-exactness properties from Theorem $1.3(\mathrm{a} 2)$ then show that $\mathbb{D}_{Y}\left(\mathbb{1}_{Y}(p)[2 p]\right)$ is itself of weight zero. Again by Theorem 1.3 (a2), the same is true for its image under $f_{!}$.

q.e.d.

\section{Definition of the motivic intersection com- plex}

Fix a scheme $X$. Since (by the conventions fixed in the beginning) $X$ is excellent, there is an open immersion $j: U \hookrightarrow X$ whose image $U$ is dense in $X$, and regular. Recall that by Theorem 1.3 (a1), the Beilinson motive $\mathbb{1}_{U}$ 
belongs to $C H M(U)_{\mathbb{Q}}$, and that by Theorem 1.7 (a), it can be extended to $C H M(X)_{\mathbb{Q}}$.

Definition 2.1. A pair $\left(j_{! *} \mathbb{1}_{U}, \alpha\right)$ is called motivic intersection complex on $X$ if the following conditions are satisfied.

(1) The object $j_{! *} \mathbb{1}_{U}$ belongs to $C H M(X)_{\mathbb{Q}}$, and

$$
\alpha: j^{*} j_{! *} \mathbb{1}_{U} \stackrel{\sim}{\longrightarrow} \mathbb{1}_{U}
$$

is an isomorphism in $C H M(U)_{\mathbb{Q}}$.

(2) The morphism induced by $\alpha$,

$$
j^{*}: \operatorname{End}_{C H M(X)_{\mathbb{Q}}}\left(j_{! *} \mathbb{1}_{U}\right) \longrightarrow \operatorname{End}_{C H M(U)_{\mathbb{Q}}}\left(\mathbb{1}_{U}\right)
$$

is injective.

Given that $j^{*}$ is full (Theorem [1.7), axiom (2) is equivalent to requiring the restriction from $\operatorname{End}_{C H M(X)_{\mathbb{Q}}}\left(j_{!_{*}} \mathbb{1}_{U}\right)$ to $\operatorname{End}_{C H M(U)_{\mathbb{Q}}}\left(\mathbb{1}_{U}\right)$ to be bijective.

Denote by $i$ the closed immersion of the complement $Z$ (with the reduced structure, say) into $X$.

Remark 2.2. When $S=\operatorname{Spec} k$ for a finite field $k$ of characteristic $p$, let us consider the formalism of weights on perverse $\ell$-adic sheaves, for $\ell \neq p$ [BBD, Sect. 5].

(a) One of the main results from [loc. cit.] states that $j_{! *}$ is a functor which transforms perverse sheaves which are pure of a given weight into perverse sheaves which are pure of the same weight $[\mathrm{BBD}$, Cor. 5.4.3]. In particular, the intersection complex $j_{! *} \mathbb{Q}_{\ell}$ is indeed pure of weight 0 .

(b) Localization implies that the kernel of

$$
j^{*}: \operatorname{End}_{X}\left(j_{! *} \mathbb{Q}_{\ell}\right) \longrightarrow \operatorname{End}_{U}\left(\mathbb{Q}_{\ell}\right)
$$

is a quotient of the group

$$
\operatorname{Hom}_{Z}\left(i^{*} j_{! *} \mathbb{Q}_{\ell}, i^{!} j_{! *} \mathbb{Q}_{\ell}\right) .
$$

But this group is zero since with respect to the perverse $t$-structure, the object $i^{*} j_{! *} \mathbb{Q}_{\ell}$ in concentrated in degrees $\leq-1$, while $i^{!} j_{! *} \mathbb{Q}_{\ell}$ is in degrees $\geq 1$ [BBD, Cor. 1.4.25].

Since the (perverse) $t$-structure for Beilinson motives is not known to exist in general, the naïve generalization of the definition of the intersection complex is not possible (but see [Sb, Sect. 3] for the case of Artin-Tate motives over a number ring). Definition 2.1 circumvents this problem by replacing the use of a $t$-structure by the use of the motivic weight structure! 
Example 2.3. If $X$ is regular, then $\left(\mathbb{1}_{X}, \mathrm{id}\right)$ is a motivic intersection complex, as follows from the relation to $K$-theory (see Section 1, point (A)), and from the invariance under passage from $X$ to its reduced structure $X_{\text {red }}$ (see Section 1, point (E)). Indeed, the restriction

$$
j^{*}: \operatorname{End}_{C H M(X)_{\mathbb{Q}}}\left(\mathbb{1}_{X}\right) \longrightarrow \operatorname{End}_{C H M(U)_{\mathbb{Q}}}\left(\mathbb{1}_{U}\right)
$$

then corresponds to

$$
j^{*}: \operatorname{Gr}_{\gamma}^{0} K_{0}\left(X_{\text {red }}\right)_{\mathbb{Q}} \longrightarrow \operatorname{Gr}_{\gamma}^{0} K_{0}\left(U_{\text {red }}\right)_{\mathbb{Q}} .
$$

The latter is an isomorphism since both sides are canonically isomorphic (via the rank) to $r$ copies of $\mathbb{Q}$, where $r$ is the number of connected components of $X$, which coincides with the number of connected components of $U$ (recall that $U$ is dense in $X$ ).

The same argument shows the following.

Proposition 2.4. The motivic intersection complex does not depend on the choice of dense open regular sub-scheme of $X$. More precisely, if $V$ is a dense open regular sub-scheme of $X$ contained in $U$, and if $\left(j_{! *} \mathbb{1}_{U}, \alpha\right)$ is a motivic intersection complex with respect to $U$, then

$$
\left(j_{! *} \mathbb{1}_{U}, \alpha_{\mid V}\right)
$$

is a motivic intersection complex with respect to $V$.

The proof of the following requires more efforts.

Proposition 2.5. The motivic intersection complex is compatible with restriction to open sub-schemes $W$ of $X$. More precisely, if $\left(j_{!_{*}} \mathbb{1}_{U}, \alpha\right)$ is a motivic intersection complex on $X$, then

$$
\left(\left(j_{! *} \mathbb{1}_{U}\right)_{\mid W}, \alpha_{\mid W \cap U}\right)
$$

is a motivic intersection complex on $W$.

Proof. Assume first that $W$ is dense in $X$. By Proposition 2.4, we may assume $U$ to be contained in $W$. Let

$$
\beta_{W}:\left(j_{! *} \mathbb{1}_{U}\right)_{\mid W} \longrightarrow\left(j_{! *} \mathbb{1}_{U}\right)_{\mid W}
$$

be an endomorphism restricting trivially to $U$. The inverse image from $X$ to $W$ is full (Theorem 1.7 (b)), therefore $\beta_{W}$ is the restriction to $W$ of an endomorphism $\beta$ of $j_{! *} \mathbb{1}_{U}$. By assumption, we have $j^{*} \beta=0$. Condition (2) of Definition 2.1 implies that $\beta=0$. Hence $\beta_{W}=0$.

In the general case, we follow an argument due to G. Ancona [An]. First, choose an open sub-scheme $W^{\prime}$ of $X$, contained in the complement of $W$, and such that $W \amalg W^{\prime}$ is dense in $X$. By the above, the restriction to $W \amalg W^{\prime}$ of $j_{!_{*}} \mathbb{1}_{U}$ is a motivic intersection complex. We are thus reduced to the case where $X=W \coprod W^{\prime}$. We leave it to the reader to show that the restrictions to $W$ and $W^{\prime}$ of $j_{! *} \mathbb{1}_{U}$ are then motivic intersection complexes on $W$ and $W^{\prime}$, respectively.

q.e.d. 


\section{Basic properties}

We keep the previous setting. Thus, $X$ is fixed scheme, and $j: U \hookrightarrow X$ the immersion of a dense open regular sub-scheme. The complementary immersion is denoted by $i: Z \hookrightarrow X$.

Theorem 3.1. (a) The motivic intersection complex is unique up to unique isomorphism.

(b) If the motivic intersection complex $\left(j_{!_{*}} \mathbb{1}_{U}, \alpha\right)$ exists, and if

$$
\beta: j^{*} M \stackrel{\sim}{\longrightarrow} \mathbb{1}_{U}
$$

is an isomorphism in $C H M(U)_{\mathbb{Q}}$, with $M \in C H M(X)_{\mathbb{Q}}$, then $j_{!_{*}} \mathbb{1}_{U}$ is (in general non-canonically) a direct factor of $M$. More precisely, there is an isomorphism

$$
M \stackrel{\sim}{\longrightarrow} j_{! *} \mathbb{1}_{U} \oplus i_{*} L_{Z}
$$

restricting to $\alpha^{-1} \circ \beta$ on $U$, with $L_{Z} \in C H M(Z)_{\mathbb{Q}}$.

Proof. Recall that the inverse image $j^{*}$ is full on $C H M(\bullet)$ (Theorem 1.7 (b)). Therefore, there exist morphisms of Chow motives

$$
\varphi: j_{! *} \mathbb{1}_{U} \longrightarrow M \text { and } \psi: M \longrightarrow j_{! *} \mathbb{1}_{U}
$$

extending $\beta^{-1} \circ \alpha$ and $\alpha^{-1} \circ \beta$, respectively. Observe that the composition $\psi \circ \varphi$ restricts to the identity on $\mathbb{1}_{U}$. Injectivity of

$$
j^{*}: \operatorname{End}_{C H M(X)_{\mathbb{Q}}}\left(j !_{*} \mathbb{1}_{U}\right) \longrightarrow \operatorname{End}_{C H M(U)_{\mathbb{Q}}}\left(\mathbb{1}_{U}\right)
$$

therefore implies that $\psi \circ \varphi=\mathrm{id}_{j ! * \mathbb{1}}$.

Similarly, $\varphi \circ \psi=\operatorname{id}_{M}$ if $(M, \beta)$ is another choice of motivic intersection complex; note that in this case, the relations $\psi \circ \varphi=\operatorname{id}_{j_{*} \mathbb{1}_{U}}$ and $\varphi \circ \psi=\mathrm{id}_{M}$ hold for any choices of $\varphi, \psi$, meaning that they are actually unique.

In the general case, $\varphi \circ \psi$ is an idempotent endomorphism of $M$. Since its restriction to $U$ is the identity, localization (see Section 1, point (E)) shows that its kernel is necessarily a Chow motive of the form $i_{*} L_{Z}$. The Beilinson motive $L_{Z} \in D M_{\mathrm{L}, c}(Z)$ equals both $i^{*} i_{*} L_{Z}$ and $i^{i} i_{*} L_{Z}$. By Theorem 1.3 (a2), it is of weight zero, hence a Chow motive over $Z$.

q.e.d.

Remark 3.2. In the context of perverse $\ell$-adic sheaves over schemes of finite type over a finite field, the analogue of Theorem 3.1 (b) (concerning pure complexes $M$ of $\ell$-adic sheaves on $X$ ) is a consequence of the Decomposition Theorem BBD, Thm. 5.4.5]. As illustrated by our proof, the formalism of weight structures yields a structural reason for the non-canonicity of the isomorphism of [loc. cit.].

According to Theorem 3.1(b), the motivic intersection complex (provided it exists) is indeed minimal among all possible extensions of $\mathbb{1}_{U}$ to a Chow motive over $X$. Furthermore, our result suggests a possible strategy for 
its construction: first, use Theorem 1.7 (a) to choose any extension $M \in$ $C H M(X)_{\mathbb{Q}}$ of $\mathbb{1}_{U}$; then, choose idempotent endomorphisms of $M$ to split off direct factors of the shape $i_{*} L_{Z}$, until no such factor is left. Note that it is not clear that the result is independent of the choices (of $M$ and of the splittings) made in this process. Nor is it clear that the result actually satisfies axiom (2) of Definition 2.1. We plan to elaborate on this elsewhere.

Remark 3.3. Let $X=X_{1} \cup X_{2}$ be a covering by two dense open subschemes, and assume that the motivic intersection complexes on $X_{1}$ and on $X_{2}$ exist. Using Proposition 2.5, Theorem 3.1 and Theorem 1.7, one can show that they can be glued along $X_{1} \cap X_{2}$ to give $(M, \alpha)$, with $M \in C H M(X)_{\mathbb{Q}}$, and

$$
\alpha: j^{*} M \stackrel{\sim}{\longrightarrow} \mathbb{1}_{U}
$$

Problem 3.4. In the situation of Remark 3.3, show that $(M, \alpha)$ satisfies axiom (2) of Definition 2.1.

There is one specific case where we know the solution to Problem 3.4. It is worthwhile to spell it out.

Corollary 3.5. Assume that $X$ is regular, and that $M \in D M_{\mathrm{E}}(X)$ is Nisnevich-locally isomorphic to $\mathbb{1}_{X}$, i.e., there is a finite Nisnevich covering of $X$ by schemes $U_{n}$ such that $M_{\mid U_{n}} \cong \mathbb{1}_{U_{n}}$ for all $n$. Then $X \cong \mathbb{1}_{X}$.

Proof. The separation property of $D M_{\mathrm{5}}(\bullet)[\mathrm{CD}$, Def. 2.1.11] and the $w$-exactness properties from Theorem 1.3 (a2) allow to control the weights of $M$ locally for the smooth topology; in particular, our assumptions imply that $M \in C H M(X)_{\mathbb{Q}}$. The given covering of $X$ can be refined to construct a dense open sub-scheme $j: U \hookrightarrow X$ and an isomorphism

$$
\beta: j^{*} M \stackrel{\sim}{\longrightarrow} \mathbb{1}_{U}
$$

By Example 2.3 and Theorem 3.1 (b),

$$
M \cong \mathbb{1}_{X} \oplus i_{*} L_{Z}
$$

for some $L_{Z} \in C H M(Z)_{\mathbb{Q}}$. On each $U_{n}$, the restriction

$$
\mathbb{1}_{U_{n}} \oplus\left(i_{*} L_{Z}\right)_{\mid U_{n}}
$$

has the same endomorphisms as $\mathbb{1}_{U_{n}}$. Therefore, $\left(i_{*} L_{Z}\right)_{\mid U_{n}}=0$. Separation implies that $i_{*} L_{Z}=0$.

q.e.d.

Note that if $M$ is Zariski-locally isomorphic to $\mathbb{1}_{X}$, then separation can be replaced by an application of the two localization triangles.

Remark 3.6. Corollary 3.5 and [CD, Prop. 14.3.29 (i)] can be employed to show that on a regular scheme $X$, two $\otimes$-invertible objects of $D M_{\mathrm{E}, c}(X)$ are isomorphic as soon as they are Nisnevich-locally isomorphic. 
Corollary 3.7 (Absolute purity). Let $a: X \rightarrow S^{\prime}$ be a morphism of pure relative dimension d: for any irreducible component of $S^{\prime}$ of dimension $n$, its pre-image under $a$ is of pure dimension $n+d$. Assume that both $X$ and $S^{\prime}$ are regular. Then there is an isomorphism

$$
a^{!} \mathbb{1}_{S^{\prime}}(-d)[-2 d] \cong \mathbb{1}_{X} .
$$

Proof. $\quad$ Cover $X$ by open sub-schemes which are quasi-projective over $S^{\prime}$. The discussion from point (D) of Section 10 then shows that the assumption of Corollary 3.5 is satisfied (even Zariski-locally) for $M=a^{!} \mathbb{1}_{S^{\prime}}(-d)[-2 d]$.

q.e.d.

Let us come back to the general situaton, i.e., drop the regularity assumption on $X$. We aim at a motivic analogue of [BBD, Prop. 2.1.17] which states that $j_{!_{*}} \mathcal{F}$ is auto-dual on $X$ provided that $\mathcal{F}$ is auto-dual on $U$. In order to have the motivic analogue of that assumption satisfied for $\mathbb{1}_{U}$, we suppose that the structure morphism of $X$ factors over a morphism $a: X \rightarrow S^{\prime}$ with regular target. We also suppose that $a$ is of pure relative dimension $d$. Put

$$
R:=a^{!} \mathbb{1}_{S^{\prime}}(-d)[-2 d] \in D M_{\mathrm{Б}, c}(X),
$$

and form the local duality functor $\mathbb{D}_{X}$ with respect to this choice of $R$. By absolute purity (Corollary 3.7), there is an isomorphism

$$
\gamma: \mathbb{1}_{U} \stackrel{\sim}{\longrightarrow} j^{*} R \text {. }
$$

We thus have

$$
\mathbb{D}_{X}=\underline{\operatorname{Hom}}_{X}(\bullet, R),
$$

and composition with $\gamma$ is an isomorphism of functors

$$
\underline{\operatorname{Hom}}_{U}\left(\bullet, \mathbb{1}_{U}\right) \stackrel{\sim}{\sim} \underline{\operatorname{Hom}}_{U}\left(\bullet, j^{*} R\right)=\mathbb{D}_{U} .
$$

When evaluated on $\mathbb{1}_{U}$, this gives

$$
\gamma_{*}: \mathbb{1}_{U}=\underline{\operatorname{Hom}}_{U}\left(\mathbb{1}_{U}, \mathbb{1}_{U}\right) \stackrel{\sim}{\longrightarrow} \mathbb{D}_{U}\left(\mathbb{1}_{U}\right) ;
$$

it is in this precise sense that $\mathbb{1}_{U}$ is auto-dual. Theorem 3.1 has the following formal consequence.

Corollary 3.8 (Auto-duality). If the motivic intersection complex exists, then it is auto-dual. More precisely, there is a unique isomorphism

$$
j_{! *} \mathbb{1}_{U} \stackrel{\sim}{\longrightarrow} \mathbb{D}_{X}\left(j_{! *} \mathbb{1}_{U}\right)
$$

compatible with $\alpha$ and $\gamma_{*}$ in the sense that its restriction to $U$ equals the composition

$$
\mathbb{D}(\alpha) \circ \gamma_{*} \circ \alpha: j^{*} j_{! *} \mathbb{1}_{U} \stackrel{\sim}{\longrightarrow} \mathbb{D}_{U}\left(j^{*} j_{! *} \mathbb{1}_{U}\right) .
$$

Proof. $\quad$ By Theorem $1.12(\mathrm{c}), \mathbb{D}_{X}\left(j_{! *} \mathbb{1}_{U}\right)$ is a Chow motive over $X$. Define

$$
\beta:=\gamma_{*}^{-1} \circ \mathbb{D}\left(\alpha^{-1}\right): j^{*} \mathbb{D}_{X}\left(j_{! *} \mathbb{1}_{U}\right)=\mathbb{D}_{U}\left(j^{*} j_{! *} \mathbb{1}_{U}\right) \stackrel{\sim}{\longrightarrow} \mathbb{1}_{U}
$$


With this choice, axiom (2) of Definition 2.1 is satisfied; indeed, by adjunction,

$$
\operatorname{End}_{C H M(X)_{\mathbb{Q}}}\left(\mathbb{D}_{X}\left(j_{! *} \mathbb{1}_{U}\right)\right)=\operatorname{Hom}_{C H M(X)_{\mathbb{Q}}}\left(j_{! *} \mathbb{1}_{U}, \mathbb{D}_{X}^{2}\left(j_{! *} \mathbb{1}_{U}\right)\right),
$$

and $\operatorname{id}_{X}=\mathbb{D}_{X}^{2}$ on $C H M(X)_{\mathbb{Q}}$. Our claim follows from Theorem 3.1 (a).

Definition 3.9. Assume that the motivic intersection complex $\left(j_{! *} \mathbb{1}_{U}, \alpha\right)$ exists. The pairing

$$
j_{! *} \mathbb{1}_{U} \otimes_{X} j_{! *} \mathbb{1}_{U} \longrightarrow a^{!} \mathbb{1}_{S^{\prime}}(-d)[-2 d]
$$

obtained by adjunction from the auto-duality isomorphism is called the motivic intersection pairing.

By definition, the motivic intersection pairing is non-degenerate in the sense that its adjoint is an isomorphism. Applying $a_{\text {! }}$ to the first component of its source, and $a_{*}$ to the second, we get

$$
a_{!} j_{! *} \mathbb{1}_{U} \otimes_{S^{\prime}} a_{*} j_{! *} \mathbb{1}_{U},
$$

which maps (isomorphically, by the projection formula [CD, Thm. 2.4.21 (v)]) to

$$
a_{!}\left(j_{! *} \mathbb{1}_{U} \otimes_{X} a^{*} a_{*} j_{! *} \mathbb{1}_{U}\right),
$$

and finally, via the adjunction $\left(a^{*}, a_{*}\right)$, to

$$
a_{!}\left(j_{! *} \mathbb{1}_{U} \otimes_{X} j_{! *} \mathbb{1}_{U}\right) .
$$

Composition with $a_{\text {! }}$ of the intersection pairing, and application of the adjunction $\left(a_{!}, a^{!}\right)$yields the pairing

$$
a_{!} j_{! *} \mathbb{1}_{U} \otimes_{S^{\prime}} a_{*} j_{! *} \mathbb{1}_{U} \longrightarrow \mathbb{1}_{S^{\prime}}(-d)[-2 d] .
$$

It is non-degenerate since by construction, its adjoint is the isomorphism

$$
a_{!} j_{! *} \mathbb{1}_{U} \stackrel{\sim}{\longrightarrow} \mathbb{D}_{S^{\prime}} a_{*} j_{! *} \mathbb{1}_{U}
$$

obtained from $a_{!}$of auto-duality and the formula $\mathbb{D}_{S^{\prime}} a_{*}=a_{!} \mathbb{D}_{X}$ (see Section 1 , point (D)). In particular, we get the motivic analogue of Poincaré duality for intersection cohomology.

Corollary 3.10. Assume that the motivic intersection complex $\left(j_{! *} \mathbb{1}_{U}, \alpha\right)$ exists, and that the morphism $a: X \rightarrow S^{\prime}$ is proper. Then $a_{!} j_{! *} \mathbb{1}_{U}$ is autodual.

Note that under the assumptions of Corollary 3.10 , the object $a_{!} j_{! *} \mathbb{1}_{U}$ is a Chow motive over $S^{\prime}$ (Theorem 1.3 (a2)).

Here are the few cases where we actually know the hypotheses of Theorem 3.1 (b) and Corollaries 3.8 and 3.10 to be satisfied. 
Theorem 3.11. The motivic intersection complex exists in the following cases.

(a) The normalization $X^{\text {norm }}$ of the reduced scheme underlying $X$ is regular.

(b) $X$ is of dimension at most two, and the residue fields of the singular points of $X^{\text {norm }}$ are perfect.

The proof of Theorem 3.11 will be given in the next section.

Remark 3.12. Let us discuss the case $S=\operatorname{Spec} \mathbb{C}$. We consider the Hodge theoretic realization

$$
R: D M_{\mathrm{E}, c}(\mathbf{S p e c} \mathbb{C}) \longrightarrow D^{b}\left(\mathbf{M H S}_{\mathbb{Q}}\right)
$$

([Hu, Sect. 2.3 and Corrigendum]; see [DG, Sect. 1.5] for a simplification of this approach). Here, $D^{b}\left(\mathbf{M H S}_{\mathbb{Q}}\right)$ is the bounded derived category of the Abelian category $\mathbf{M H S} \mathbf{S}_{\mathbb{Q}}$ of mixed graded-polarizable $\mathbb{Q}$-Hodge structures. It is reasonable to expect the Hodge realization to extend to the relative setting, yielding exact, monoidal functors

$$
R: D M_{\mathrm{E}, c}(X) \longrightarrow D^{b}\left(\mathbf{M H M}_{\mathbb{Q}} X\right)
$$

for all schemes $X$ over $\mathbb{C}$. Here, $D^{b}\left(\mathbf{M H M}_{\mathbb{Q}} X\right)$ is the bounded derived category of algebraic mixed $\mathbb{Q}$-Hodge modules on $X[\mathrm{Sa}$. Let us assume such an extension $R$ to exist, and to be compatible with the six operations from CD and from $[\mathrm{Sa}]$. According to $[\mathrm{Bo3}$, Prop. $2.7 \mathrm{I}]$, the category $D^{b}\left(\mathbf{M H M}_{\mathbb{Q}} X\right)$ carries a weight structure, with $D^{b}\left(\mathbf{M H M}_{\mathbb{Q}} X\right)_{w<0}$ and $D^{b}\left(\mathbf{M} \mathbf{H} \mathbf{M}_{\mathbb{Q}} X\right)_{w>0}$ equal to the sub-categories of complexes of Hodge modules of weights $\leq 0$ and $\geq 0$ (in the sense of [Sa, Def. 4.5]), respectively. The Hodge theoretic realization is then necessarily $w$-exact: indeed, since $D M_{\mathrm{Б}, c}(X)$ is generated by its heart (Remark 1.6), it suffices to show that Chow motives over $X$ are mapped to Hodge modules which are pure of weight zero. This in turn follows from the explicit description of $C H M(X)_{\mathbb{Q}}$, and from the $w$-exactness properties of the six operations on algebraic Hodge modules: any Chow motive over $X$ is a direct factor of

$$
f_{!} \mathbb{1}_{Y}(p)[2 p],
$$

for a proper morphism $f: Y \rightarrow X$ with regular source $Y$, and an integer $p$ (Remark 1.6). Its image under $R$ is therefore a direct factor of

$$
f_{!} \mathbb{Q}_{Y}^{H}(p)[2 p] .
$$

The $\mathbb{C}$-scheme $Y$ is regular, hence smooth over $\mathbb{C}$. By [Sa, Thm. 3.27], the variation of Hodge structure $\mathbb{Q}_{Y}^{H}(p)$ on $Y$ is a complex of algebraic Hodge modules; as such, it is pure of weight $-2 p$. Therefore, its shift $\mathbb{Q}_{Y}^{H}(p)[2 p]$ is pure of weight zero. But by $\left[\mathrm{Sa}\right.$, Sect. (4.5.2)], $f_{!}=f_{*}$ is $w$-exact.

The canonical $t$-structure on $D^{b}\left(\mathbf{M H M}_{\mathbb{Q}} X\right)$ allows to define the Hodge theoretic intersection complex $I C_{X} \mathbb{Q}^{H}$ on $X$ [Sa, Sect. 4.5]. Due to the normalization we chose for the motivic intersection complex, we define

$$
j_{! *} \mathbb{Q}_{U}^{H}:=I C_{X} \mathbb{Q}^{H}[-d]
$$


if $X$ is of pure dimension $d$. (Thus, $j_{!_{*}} \mathbb{Q}_{U}^{H}$ is a complex of Hodge modules concentrated in degree $d$.) According to [Sa, Sect. 4.5], $j_{! *} \mathbb{Q}_{U}^{H}$ is pure of weight zero, and extends $\mathbb{Q}_{U}^{H}$. It satisfies the Hodge theoretic analogue of axiom (2) of Definition 2.1. From the Hodge theoretic analogue of Theorem 3.1 (b), we conclude that the realization $R\left(j_{! *} \mathbb{1}_{U}\right)$ of the motivic intersection complex contains $j_{! *} \mathbb{Q}_{U}^{H}$ as a direct factor.

When $S=$ Spec $k$ is the spectrum of a finite field $k$, similar remarks apply to perverse $\ell$-adic sheaves over $k$-schemes.

Problem 3.13. In the situation of Remark 3.12, show the equality

$$
R\left(j_{!_{*}} \mathbb{1}_{U}\right)=j_{! *} \mathbb{Q}_{U}^{H}
$$

Note that it implies that the intersection motive of $X$ realizes to give (the complex computing) intersection cohomology of $X$.

\section{On the problem of existence}

We keep the situation considered before: $X$ is a scheme, and $j: U \hookrightarrow X$ is the immersion of a dense open regular sub-scheme. The complementary immersion is denoted by $i: Z \hookrightarrow X$.

Proposition 4.1. Let $Y$ be a scheme, $h: V \hookrightarrow Y$ an open immersion, and $k: T \hookrightarrow Y$ the complement. Let $L$ and $N$ be objects of $D M_{\mathrm{E}}(Y)$. Assume that $\operatorname{Hom}_{T}\left(k^{*} L, k^{!} N\right)=0$. Then the restriction

$$
h^{*}: \operatorname{Hom}_{Y}(L, N) \longrightarrow \operatorname{Hom}_{V}\left(h^{*} L, h^{*} N\right)
$$

is injective. of

Proof. Either one of the localization triangles implies that the kernel

$$
h^{*}: \operatorname{Hom}_{Y}(L, N) \longrightarrow \operatorname{Hom}_{V}\left(h^{*} L, h^{*} N\right)
$$

is a quotient of $\operatorname{Hom}_{T}\left(k^{*} L, k ! N\right)$.

q.e.d.

In the setting of interest for us, Proposition 4.1 implies the following.

Corollary 4.2. Assume that $M \in C H M(X)_{\mathbb{Q}}$ is given, together with an isomorphism

$$
\alpha: j^{*} M \stackrel{\sim}{\longrightarrow} \mathbb{1}_{U}
$$

If

$$
\operatorname{Hom}_{Z}\left(i^{*} M, i^{!} M\right)=0,
$$

then $(M, \alpha)$ equals the motivic intersection complex on $X$. 
Replacing axiom (2) of Definition 2.1 by the vanishing of

$$
\operatorname{Hom}_{Z}\left(i^{*} j_{! *} \mathbb{1}_{U}, i^{!} j_{! *} \mathbb{1}_{U}\right)
$$

might possibly provide a "better" definition of the motivic intersection complex. At least, the proof of Theorem 3.11 will consist in showing this vanishing. In order to do so, the following principle will be frequently used.

Corollary 4.3. Let $Y$ be a scheme, $h: V \hookrightarrow Y$ an open immersion, and $k: T \hookrightarrow Y$ the complement. Let $L$ and $N$ be objects of $D M_{\mathrm{E}}(Y)$. If

$$
\operatorname{Hom}_{V}\left(h^{*} L, h^{*} N\right) \text { and } \operatorname{Hom}_{T}\left(k^{*} L, k ! N\right)=0 \text {, }
$$

then

$$
\operatorname{Hom}_{Y}(L, N)=0 \text {. }
$$

Successive applications of this principle show that the vanishing assumption of Corollary 4.2 can be verified on a finite stratification.

Example 4.4. We get another proof of the equality " $j_{!_{*}} \mathbb{1}_{U}=\mathbb{1}_{X}$ " for regular $X$ (Example 2.3): choose a stratification of $Z$ by regular sub-schemes $T$. Then apply absolute purity and the relation to $K$-theory to see that

$$
\operatorname{Hom}_{T}\left(i^{*} \mathbb{1}_{X}, i^{!} \mathbb{1}_{X}\right)=0
$$

for each $T$.

Let us turn to the proof of Theorem 3.11. We may assume that $X$ is reduced. Denote by

$$
p: X^{n o r m} \longrightarrow X
$$

the normalization of $X$; note that $p$ is finite since $X$ is excellent. Note also that $j$ factors uniquely through an open immersion $j^{\text {norm }}$ into $X^{\text {norm }}$, identifying $U$ with its pre-image under $p$. Part (a) of Theorem 3.11 is contained in the following.

Proposition 4.5. Assume that $X^{\text {norm }}$ is regular. Then $\left(p_{!} \mathbb{1}_{X^{\text {norm }}}\right.$, id $)$ equals the motivic intersection complex on $X$.

Proof. First, note that $X^{\text {norm }}$ being supposed regular, the Beilinson motive $\mathbb{1}_{X^{\text {norm }}}$ is indeed a Chow motive (Theorem 1.3 (a1)). Since $p_{!}=p_{*}$, the same is true for $p_{!} \mathbb{1}_{X^{\text {norm }}}$ (Theorem 1.3 (a2)). Define $X^{n n}:=$ $X^{\text {norm }} \times_{X} X^{\text {norm }}$, denote by $p_{1}$ and $p_{2}$ the projections of $X^{n n}$ to the two factors $X^{\text {norm }}$, and by $P$ the projection to $X$. Base change (see Section 1, point (B)) and adjunction, first from $Z$ to $p^{-1}(Z)$ and then to $P^{-1}(Z)$, show that

$$
\operatorname{Hom}_{Z}\left(i^{*} p_{!} \mathbb{1}_{X^{n o r m}}, i^{!} p_{!} \mathbb{1}_{X^{n o r m}}\right)=\operatorname{Hom}_{P^{-1}(Z)}\left(i^{n n, *} p_{2}^{*} \mathbb{1}_{X^{n o r m}}, i^{n n, !} p_{1}^{!} \mathbb{1}_{X^{n o r m}}\right),
$$

where we let $i^{n n}$ denote the immersion of $P^{-1}(Z)$ into $X^{n n}$. Let $k: T \hookrightarrow$ $P^{-1}(Z)$ be a regular connected locally closed sub-scheme. It is necessarily 
quasi-finite over $Z$. In particular, its relative dimension $e$ over $X^{\text {norm }}$ via $p_{1}$ is strictly negative. As recalled in Section 1, point (D),

$$
k^{!} i^{n n, !} p_{1}^{!} \mathbb{1}_{X^{n o r m}}=\mathbb{1}_{T}(e)[2 e] .
$$

Of course,

$$
k^{*} i^{n n, *} p_{2}^{*} \mathbb{1}_{X^{n o r m}}=\mathbb{1}_{T} .
$$

The relation to $K$-theory shows that

$$
\operatorname{Hom}_{T}\left(\mathbb{1}_{T}, \mathbb{1}_{T}(e)[2 e]\right)=0 .
$$

Indeed, the graded object $\operatorname{Gr}_{\gamma}^{e} K_{0}(T)$ is zero since the gamma filtration is concentrated in non-negative degrees. Now apply Corollaries 4.3 and 4.2 .

q.e.d.

Problem 4.6. Without the regularity assumption on $X^{\text {norm }}$, show that

$$
j_{! *} \mathbb{1}_{U}=p_{!} j_{! *}^{\text {norm }} \mathbb{1}_{U}
$$

whenever the motivic intersection complex $j_{! *}^{\text {norm }} \mathbb{1}_{U}$ on $X^{\text {norm }}$ exists.

In order to prove part (b) of Theorem 3.11, note first that for reduced schemes $X$ of dimension at most one, the normalization $X^{\text {norm }}$ is regular. For the rest of this section, let us therefore assume that $X$ is a reduced surface (i.e., all irreductible components of $X$ are integral and of dimension two), and that the residue fields of the singular points of $X^{\text {norm }}$ are perfect.

Let us start by the construction of $j_{! *}^{\text {norm }} \mathbb{1}_{U}$ on $X^{\text {norm }}$. It is a variant of the construction from [CM] for surfaces defined over a field. By Proposition 2.4, we may perform the computation after replacing $U$ by the regular locus $V$ of $X^{\text {norm }}$. Since $X^{\text {norm }}$ is regular in codimension one, the complement $Z^{\prime}$ of $V$ (with the reduced structure) is finite; in fact, by our assumption, $Z^{\prime}$ is the spectrum of a finite product of perfect fields. By Abhyankar's result on resolution of singularities in dimension two [L2, Theorem], $X$ can be desingularized. In addition (see the discussion in [L1, pp. 191-194]), by further blowing up possible singularities of (the components of) the preimage $D$ of $Z^{\prime}$, it can be assumed to be a divisor with normal crossings, whose irreducible components are regular. Fix such a resolution, that is, fix the following diagram, assumed to be cartesian:

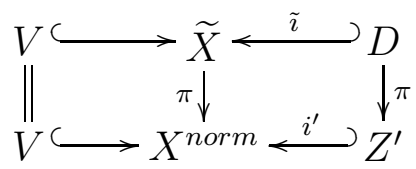

where $\pi$ is proper (and birational), $\widetilde{X}$ is regular, and $D$ is a divisor with normal crossings, whose irreducible components $D_{m}$ are regular. 
Thus, the $D_{m}$ are regular curves over perfect fields (the points of $Z^{\prime}$ ). Therefore, they are smooth. In addition, they are proper. Denote by $\tilde{\imath}_{m}$ the closed immersion of $D_{m}$ into $\widetilde{X}$, and by $\pi_{m}$ the restriction of $\pi$ to $D_{m}$. The classical theory of Chow motives yields canonical (split) sub-objects $\left(\pi_{m, !} \mathbb{1}_{D_{m}}\right)^{0}$ and (split) quotients $\left(\pi_{m, !} \mathbb{1}_{D_{m}}\right)^{2}$ of $\pi_{m, !} \mathbb{1}_{D_{m}}$. The adjunctions $\operatorname{id}_{\tilde{X}} \rightarrow \tilde{\imath}_{m, *} \tilde{l}_{m}^{*}$ and $\tilde{\imath}_{m, *} \tilde{l}_{m}^{!} \rightarrow \operatorname{id}{ }_{\tilde{X}}$, and absolute purity for $\tilde{\imath}_{m}$ yield canonical morphisms

$$
\tilde{\imath}^{*}: \pi_{!} \mathbb{1}_{\widetilde{X}} \longrightarrow \bigoplus_{m} \pi_{m, !} \mathbb{1}_{D_{m}} \longrightarrow \bigoplus_{m}\left(\pi_{m, !} \mathbb{1}_{D_{m}}\right)^{2}
$$

and

$$
\tilde{\imath}_{*}: \bigoplus_{m}\left(\pi_{m, !} \mathbb{1}_{D_{m}}\right)^{0}(-1)[-2] \hookrightarrow \bigoplus_{m} \pi_{m, !} \mathbb{1}_{D_{m}}(-1)[-2] \longrightarrow \pi_{!} \mathbb{1}_{\tilde{X}}
$$

of Chow motives over $X^{\text {norm }}$.

Proposition 4.7. (i) The composition $\alpha:=\tilde{\imath}^{*} \tilde{\imath}_{*}$ is an isomorphism.

(ii) The composition $\varepsilon:=\tilde{\imath}_{*} \alpha^{-1} \tilde{\imath}^{*}$ is an idempotent on $\pi_{!} \mathbb{1}_{\tilde{X}}$. Hence so is the difference $\operatorname{id}_{\pi ! \mathbb{1}_{\tilde{X}}}-\varepsilon$.

(iii) The image im $\varepsilon$ is canonically isomorphic to $\oplus_{m}\left(\pi_{m, !} \mathbb{1}_{D_{m}}\right)^{2}$.

Proof. The proof is formally identical to the one of [W2, Thm. 2.2]. Observe that the non-degeneracy of the intersection pairing on the components of $D$ holds since the proof [M, p. 6] carries over to the general context of normal surfaces.

q.e.d.

Note that the $\left(\pi_{m, !} \mathbb{1}_{D_{m}}\right)^{2}$ restrict trivially to $V$. Therefore, the image $\operatorname{im}\left(\operatorname{id}_{\pi ! \mathbb{1}_{\tilde{x}}}-\varepsilon\right)$ restricts to give $\mathbb{1}_{V}$. Part (b) of Theorem 3.11 is contained in the following.

Proposition 4.8. Assume that $X$ is a surface, and that the residue fields of the singular points of $X^{\text {norm }}$ are perfect.

(a) The pair $\left(\operatorname{im}_{\left(\mathrm{id}_{\pi !} \mathbb{1}_{\tilde{X}}\right.}-\varepsilon\right)$,id) equals the motivic intersection complex on $X^{\text {norm }}$.

(b) The pair $\left(p_{!} \operatorname{im}\left(\mathrm{id}_{\pi !} \mathbb{1}_{\tilde{X}}-\varepsilon\right)\right.$, id) equals the motivic intersection complex on $X$.

Proof. It suffices to prove part (b). Write $M:=\operatorname{im}\left(\mathrm{id}_{\pi_{!} \mathbb{1}_{\tilde{X}}}-\varepsilon\right)$. As in the proof of Proposition 4.5, let $X^{n n}=X^{n o r m} \times_{X} X^{n o r m}$, denote by $p_{1}$ and $p_{2}$ the projections of $X^{n n}$ to the two factors $X^{\text {norm }}$, and by $P$ the projection to $X$. Base change and adjunction show that

$$
\operatorname{Hom}_{Z}\left(i^{*} p_{!} M, i^{!} p_{!} M\right)=\operatorname{Hom}_{P^{-1}(Z)}\left(i^{n n, *} p_{2}^{*} M, i^{n n, !} p_{1}^{!} M\right),
$$

where $i^{n n}$ denotes the immersion of $P^{-1}(Z)$ into $X^{n n}$. In order to apply Corollary 4.2, we need to show the vanishing of this group. We shall repeatedly apply Corollary 4.3. In order to do so, stratify $P^{-1}(Z)$ as follows: the open 
stratum (possibly empty) is the intersection of the pre-images under $p_{1}$ and under $p_{2}$ of $V$ (which contains $U$ ), the closed stratum is the complement, which is a finite set of points.

If $k: T \hookrightarrow P^{-1}(Z)$ is a regular connected locally closed sub-scheme of the open stratum, then its relative dimension $e$ over $X^{n o r m}$ via $p_{1}$ is strictly negative. Since $p_{1}(T)$ and $p_{2}(T)$ are contained in $V$, and $\operatorname{im}\left(\operatorname{id}_{\pi !} \mathbb{1}_{\tilde{x}}-p\right)$ restricts to $\mathbb{1}_{V}$, we argue as in the proof of Proposition 4.5 to see that

$$
\operatorname{Hom}_{T}\left(k^{*} i^{n n, *} p_{2}^{*} M, k^{!} i^{n n, !} p_{1}^{!} M\right)=0 .
$$

It remains to check the points $k: T \hookrightarrow P^{-1}(Z)$ of the closed stratum. Depending on whether $p_{2}(T)$ is regular or not, we have

$$
k^{*} i^{n n, *} p_{2}^{*} M=\mathbb{1}_{T}
$$

or

$$
k^{*} i^{n n, *} p_{2}^{*} M=\left(\pi_{!} \mathbb{1}_{D_{p_{2}(T)}}\right)^{\leq 1},
$$

where $D_{p_{2}(T)}$ is the base change of the exceptional divisor $D$ to $p_{2}(T)$, and where the symbol $\left(\pi_{!} \mathbb{1}_{D_{p_{2}(T)}}\right)^{\leq 1}$ denotes the kernel of the projection

$$
\pi_{!} \mathbb{1}_{D_{p_{2}(T)}} \longrightarrow\left(\pi_{!} \mathbb{1}_{D_{p_{2}(T)}}\right)^{2}:=\bigoplus_{m}\left(\pi_{m, !} \mathbb{1}_{D_{m, p_{2}(T)}}\right)^{2}
$$

induced by $\varepsilon$. Similarly (see Section 1, point (D)),

$$
k^{!} i^{n n, !} p_{1}^{!} M=\mathbb{1}_{T}(-2)[-4]
$$

or

$$
k^{!} i^{n n, !} p_{1}^{!} M=\left(\pi_{!}\left(\tilde{\imath}_{\mid D_{p_{1}(T)}}\right)^{!} \mathbb{1}_{\tilde{X}}\right)^{\leq 1} .
$$

We thus need to distinguish four cases. As usual,

$$
\operatorname{Hom}_{T}\left(\mathbb{1}_{T}, \mathbb{1}_{T}(-2)[-4]\right)=0 .
$$

In order to show that

$$
\operatorname{Hom}_{T}\left(\mathbb{1}_{T},\left(\pi_{!}\left(\tilde{l}_{\mid D_{p_{1}(T)}}\right)^{!} \mathbb{1}_{\tilde{X}}\right)^{\leq 1}\right)=0,
$$

note that $\pi_{!}=\pi_{*}$, and that by adjunction,

$$
\operatorname{Hom}_{T}\left(\mathbb{1}_{T}, \pi_{*}\left(\tilde{l}_{\left.\mid D_{p_{1}(T)}\right)}\right)^{!} \mathbb{1}_{\widetilde{X}}\right)=\operatorname{Hom}_{D_{p_{1}(T)}}\left(\mathbb{1}_{D_{p_{1}(T)}},\left(\tilde{\imath}_{\mid D_{p_{1}(T)}}\right)^{!} \mathbb{1}_{\widetilde{X}}\right) .
$$

Stratify $D_{p_{1}(T)}$ by regular sub-schemes, using that $\tilde{\imath}$ is of stricly positive codimension, to see that the latter group is zero.

Similarly,

$$
\operatorname{Hom}_{T}\left(\left(\pi_{!} \mathbb{1}_{D_{p_{2}(T)}}\right)^{\leq 1}, \mathbb{1}_{T}(-2)[-4]\right)=0 .
$$

It remains to consider

$$
\operatorname{Hom}_{T}\left(\left(\pi_{!} \mathbb{1}_{D_{p_{2}(T)}}\right)^{\leq 1},\left(\pi_{!}\left(\tilde{\imath}_{\mid D_{p_{1}(T)}}\right)^{!} \mathbb{1}_{\widetilde{X}}\right)^{\leq 1}\right) .
$$


Adjunction yield an identification between

$$
\operatorname{Hom}_{T}\left(\pi_{!} \mathbb{1}_{D_{p_{2}(T)}}, \pi_{!}\left(\tilde{\imath}_{\mid D_{p_{1}(T)}}\right) ! \mathbb{1}_{\tilde{X}}\right)
$$

and

$$
\operatorname{Hom}_{D_{p_{2}(T)} \times{ }_{T} D_{p_{1}(T)}}\left(\mathbb{1}_{D_{p_{2}(T)} \times{ }_{T} D_{p_{1}(T)}}, p r_{D_{p_{1}(T)}}^{!}\left(\tilde{l}_{\mid D_{p_{1}(T)}}\right) ! \mathbb{1}_{\tilde{X}}\right),
$$

where $D_{p_{2}(T)} \times_{T} D_{p_{1}(T)}$ is the (singular) surface obtained by base change over $T$ of the curves $D_{p_{2}(T)}$ and $D_{p_{1}(T)}$, and $p r_{D_{p_{1}(T)}}$ denotes the projection to $D_{p_{1}(T)}$. On regular sub-schemes contained in the singular locus of $D_{p_{2}(T)} \times_{T}$ $D_{p_{1}(T)}$, the same considerations as before show that there are no non-zero morphisms of the required type. Hence the assumption of Proposition 4.1 is satisfied, and

$$
\operatorname{Hom}_{D_{p_{2}(T)} \times{ }_{T} D_{p_{1}(T)}}\left(\mathbb{1}_{D_{p_{2}(T)} \times{ }_{T} D_{p_{1}(T)}}, \operatorname{pr}_{D_{p_{1}(T)}}^{!}\left(\tilde{l}_{\mid D_{p_{1}(T)}}\right) ! \mathbb{1}_{\tilde{X}}\right)
$$

injects into

$$
\operatorname{Hom}_{D_{p_{2}(T)_{\text {reg }}} \times_{T} D_{p_{1}(T)_{\text {reg }}}}\left(\mathbb{1}_{D_{p_{2}(T)}{ }_{\text {reg }} \times_{T} D_{p_{1}(T)_{\text {reg }}}}, \operatorname{pr}_{D_{p_{1}(T)_{\text {reg }}}}^{l_{l}}\left(\tilde{l}_{\mid D_{p_{1}(T)_{\text {reg }}}}\right) ! \mathbb{1}_{\tilde{X}}\right),
$$

where the subscripts reg denote the regular loci. Both $D_{p_{2}(T)_{r e g}} \times_{T} D_{p_{1}(T)_{r e g}}$ and $\widetilde{X}$ are regular surfaces, hence

$$
\operatorname{pr}_{D_{p_{1}(T)_{r e g}}}^{!}\left(\tilde{l}_{\mid D_{p_{1}(T)} \text { reg }}\right) ! \mathbb{1}_{\widetilde{X}}=\mathbb{1}_{D_{p_{2}(T)_{\text {reg }}} \times_{T} D_{p_{1}(T)_{\text {reg }}}} .
$$

Therefore,

$$
\operatorname{Hom}_{D_{p_{2}(T)_{r e g}} \times_{T} D_{p_{1}(T)_{r e g}}}\left(\mathbb{1}_{D_{p_{2}(T)_{r e g}} \times_{T} D_{p_{1}(T)_{r e g}}}, \operatorname{pr}_{D_{p_{1}(T)_{r e g}}}^{\imath_{\mid}}\left(\tilde{l}_{\mid D_{p_{1}(T)_{r e g}}}\right) ! \mathbb{1}_{\tilde{X}}\right)
$$

equals $r$ copies of $\mathbb{Q}$, where $r$ is the number of connected components of $D_{p_{2}(T)_{r e g}} \times_{T} D_{p_{1}(T)_{r e g}}$. But the same result, with compatible identifications is obtained by computing

$$
\operatorname{Hom}_{T}\left(\left(\pi_{!} \mathbb{1}_{D_{p_{2}(T)}}\right)^{2},\left(\pi_{!}\left(\tilde{\imath}_{\mid D_{p_{1}(T)}}\right)^{!} \mathbb{1}_{\tilde{X}}\right)^{2}\right) .
$$

q.e.d.

\section{References}

[An] G. Ancona, letter to the author dated Apr. 14, 2011.

[Ay] J. Ayoub, Les six opérations de Grothendieck et le formalisme des cycles évanescents dans le monde motivique (I-II), Astérisque 314-315, Soc. Math. France (2007).

[BBD] A.A. Beilinson, J. Bernstein, P. Deligne, Faisceaux pervers, in: B. Teissier, J.L. Verdier (eds.), Analyse et topologie sur les espaces singuliers (I), Astérisque 100, Soc. Math. France (1982). 
[Bo1] M.V. Bondarko, Weight structures vs. t-structures; weight filtrations, spectral sequences, and complexes (for motives and in general), J. KTheory 6 (2010), 387-504.

[Bo2] M.V. Bondarko, Weight for relative motives; relation with mixed sheaves, preprint, July 2010, version dated Sept. 21, 2010, 42 pages, available on arXiv.org under http://arxiv.org/abs/1007.4543

[Bo3] M.V. Bondarko, Weights and t-structures: in general triangulated categories, for 1-motives, mixed motives, and for mixed Hodge complexes and modules, preprint, November 2010, version dated Mar. 28, 2011, 27 pages, available on arXiv.org under http://arxiv.org/abs/1011.3507

[CD] D.-C. Cisinski, F. Déglise, Triangulated categories of mixed motives, preprint, December 2009, 229 pages, available on arXiv.org under http://arxiv.org/abs/0912.2110

[CM] M.A.A. de Cataldo, L. Migliorini, The Chow motive of semismall resolutions, Math. Res. Lett. 11 (2004), 151-170.

[CH] A. Corti, M. Hanamura, Motivic decomposition and intersection Chow groups. I, Duke Math. J. 103 (2000), 459-522.

[DG] P. Deligne, A.B. Goncharov, Groupes fondamentaux motiviques de Tate mixte, Ann. Scient. ENS 38 (2005), 1-56.

[GHM] B.B. Gordon, M. Hanamura, J.P. Murre, Relative Chow-Künneth projectors for modular varieties, J. reine angew. Math 558 (2003), 1-14.

[Hé1] D. Hébert, Structures de poids à la Bondarko sur les motifs de Beilinson, to appear in Compositio Math., 17 pages, available on arXiv.org under http://arxiv.org/abs/1007.0219

[Hé2] D. Hébert, Complexe de Poids, Dualité et Motifs de Beilinson, preprint, October 2010, 17 pages, available on arXiv.org under http://arxiv.org/abs/1010.5469

[Hu] A. Huber, Realization of Voevodsky's motives, J. of Alg. Geom. 9 (2000), 755-799, Corrigendum, 13 (2004), 195-207.

[L1] J. Lipman, Introduction to resolution of singularities, in R. Hartshorne (ed.), Algebraic Geometry. Proceedings of the Symposium in Pure Mathematics of the AMS, held at Humboldt State University, Arcata, California, July 29-August 16, 1974, Proc. of Symp. in Pure Math. 29, AMS (1975), 187-230.

[L2] J. Lipman, Desingularization of two-dimensional schemes, Ann. of Math. 107 (1978), 151-207. 
[M] D. Mumford, The topology of normal singularities of an algebraic surface and a criterion for simplicity, Publ. Math. IHES 9 (1961), 5-22.

[Sa] M. Saito, Mixed Hodge Modules, Publ. RIMS, Kyoto Univ. 26 (1990), 221-333.

[Sb] J. Scholbach, Mixed Artin-Tate motives over number rings, preprint, March 2010, version dated Aug. 5, 2010, 28 pages, available on arXiv.org under http://arxiv.org/abs/1003.1267

[S] A.J. Scholl, Integral elements in $K$-theory and products of modular curves, in B.B. Gordon, J.D. Lewis, S. Müller-Stach, S. Saito, N. Yui (eds.), The Arithmetic and Geometry of Algebraic Cycles. Proceedings of the NATO Advanced Study Institute held as part of the 1998 CRM Summer School at Banff, Kluwer Acad. Publ. (2000), 467-489.

[VSF] V. Voevodsky, A. Suslin, E.M. Friedlander, Cycles, Transfers, and Motivic Homology Theories, Ann. of Math. Studies 143, Princeton Univ. Press 2000.

[W1] J. Wildeshaus, On the interior motive of certain Shimura varieties: the case of Hilbert-Blumenthal varieties, preprint, June 2009, version dated March 18, 2011, 31 pages, submitted, available on arXiv.org under http://arxiv.org/abs/0906.4239

[W2] J. Wildeshaus, Pure motives, mixed motives and extensions of motives associated to singular surfaces, 39 pages, to appear in M. Kim, S. Ramdorai, L. Lafforgue, A. Genestier, B.-C. Ngo (eds.), Autour des motifs. Actes de l'école d'été franco-asiatique de géométrie algébrique et théorie des nombres, IHES, Juillet 2006, Panoramas et synthèses, SMF (2011), available on arXiv.org under http://arxiv.org/abs/0706.4447 